\title{
新型冠状病毒肺炎疫情数据挖掘与离散随机传播 动力学模型分析
}

唐三一 ${ }^{1}$ ，唐彪 ${ }^{2}$, Nicola Luigi Bragazzi ${ }^{2}$ ，夏凡 ${ }^{3}$ ，李堂娟 ${ }^{3}$ ，何莎 ${ }^{1}$, 任鹏宇 ${ }^{4}$, 王霞 ${ }^{1}$, 向长城 ${ }^{5}$, 彭志行 ${ }^{6}$, 吴建宏 ${ }^{2}$, 肖燕妮 ${ }^{3 *}$

1. 陕西师范大学数学与信息科学学院, 西安 710062 ;

2. Department of Mathematics and Statistics, York University, Toronto M3J 1P3, Canada;

3. 西安交通大学数学与统计学院, 数学与生命科学交叉中心, 西安 710049 ;

4. 西安交通大学第二附属医院, 西安 710004 ;

5. 湖北民族大学数学与统计学院, 恩施 440015 ;

6. 南京医科大学公共卫生学院, 南京 211166

E-mail: sytang@snnu.edu.cn, btang66@yorku.ca, robertobragazzi@gmail.com,xiafan1995@stu.xjtu.edu.cn, litangjuan1234@stu.xjtu.edu.cn, hesha@snnu.edu.cn, renpengyu.xjut@xjtu.edu.cn, xiawang@snnu.edu.cn, xcc7426681@126.com,zhihangpeng@njme.edu.cn,wujhhida@gmail.com,yxiao@mail.xjtu.edu.cn

收稿日期: 2020-02-26；接受日期: 2020-03-02；网络出版日期: 2020-03-10；＊通信作者 国家自然科学基金 (批准号: 11631012, 61772017 和 11961024) 资助项目

摘要 新型冠状病毒肺炎 (COVID-19) 疫情已经蔓延至全国各地, 包括陕西省在内很多省份的早期疫 情均以输入病例为主, 后期的疫情在严格的防控措施下也已呈下降趋势。评价防控措施的有效性、分 析人口流动对疫情的影响对于研究陕西省 (或其他以输入病例为主的地区) 疫情和未来应对突发性传 染病有着重要的意义. 根据陕西省卫生健康委员会 (简称卫健委) 公布的详实数据信息可以挖掘传播 链 (感染树), 得到从发病到首诊、入院、确诊的中位持续时间, 每日潜伏者类、感染者类、治疗者类的 具体人数和感染者状态转移的空间分布. 本文计算确定 COVID-19 疫情的控制再生数 (1.48-1.69), 并 发展新的统计推断方法获得陕西省严控措施下的有效再生数; 进而提出一个全新的融入了公共卫生干 预和输入病例的离散随机 COVID-19 疫情传播模型, 通过多源数据实现了模型的参数化, 分析不同的 流动模式、输入人口中感染者的比例对二次暴发风险的影响. 主要结论显示, 间歇性的人口流动、密 切关注和有效隔离流动人口中的感染者能有效降低二次暴发的风险, 为有序组织复工、复学提供决策 支持.

关键词 新型冠状病毒肺炎 输入病例 离散随机模型 有效再生数 人口流动

MSC (2010) 主题分类 $34 \mathrm{~F} 05,65 \mathrm{~N} 21$

英文引用格式: Tang S Y, Tang B, Bragazzi N L, et al. Analysis of COVID-19 epidemic traced data and stochastic discrete transmission dynamic model (in Chinese). Sci Sin Math, 2020, 50: 1071-1086, doi: 10.1360/SSM-2020-0053 


\section{1 引言}

冠状病毒是一种单链 RNA 病毒, 因其呈球状且外周有花冠状刺突而得名. 它们通常会引起轻微的 呼吸道感染, 有时也会有致命威胁. 自首次发现以来, 中国、沙特阿拉伯王国和韩国分别于 2003、2012 和 2015 年发生了 3 起重大疫情. 由世界卫生组织命名的 COVID-19 引起的第 4 次大规模暴发, 由武 汉首发并快速向全国蔓延, 现已在全世界众多国家暴发 (参见文献 [1-4]). 截至 2020 年 2 月 23 日, 已 造成中国超过 77,000 人感染和 2,592 人死亡 (参见文献 [5]). 自 2020 年 1 月 23 日以来, 中国政府一 直在实施前所未有的、越来越严格的公共卫生干预措施, 包括全面封锁武汉和周边城市, 全国范围内 密切跟踪接触者和限制出行等 (参见文献 [5]). 因此, 只有发展有效方法将干预措施融入经典的流行病 模型中, 才能对 COVID-19 在武汉市或湖北省传播的流行病学特征进行可靠的估计, 并准确地预测流 行趋势和达峰时间 (参见文献 [6-10]). 近期, 很多建模研究工作主要是利用少量的数据, 侧重于分析疾 病早期传播风险 (参见文献 [11-14]). 但是, 如何根据实时更新的数据, 刻画和评估包括篎查率、确诊 率和检出率等不断加强的控制措施, 需要我们不断更新模型参数和模型设置, 以期符合我国的防控策 略并服务于防控策略的有效性和时效性等的评估.

由于数据质量的不断提高, 特别是来自中国其他省市的完整数据信息, 增强了利用模型准确评估 传染病传播风险以及公共卫生干预措施效果的能力. 由于湖北省武汉市的全面封城, 很大程度上降低 了我国其他省市的输入病例数. 也正是由于输入病例的减少, 加上各地严格的密切跟踪隔离措施, 产 生了一批高质量的疫情数据, 例如, 病例输入、症状出现、首次就诊、隔离和确诊的精确时间, 这为详 细、精准分析疫情和防控策略的有效性提供了可能. 精准详实的数据对增强模型的预测评估能力至关 重要, 能为渐进式恢复正常生活对中国其他城市可能暴发的第二次疫情风险进行有效、实时的评估 ${ }^{[15]}$. 因此, 本文的主要目标之一是发展能够刻画输入病例的随机离散模型, 融合不断加强的公共卫生干预 策略, 评估以输入病例为主、疫情并不严重地区由于人口流动可能导致二次暴发的风险.

由于陕西省卫健委公布了详实的疫情数据 ${ }^{[16]}$, 使得我们能够依据记录信息进行数据挖掘, 得到每 日潜伏者类、感染者类和治疗者类人数、感染树及续代时间. 由此得到陕西的基本再生数, 并发展文 献 $[17,18]$ 中的技巧获得陕西 COVID-19 疫情风险的有效再生数. 为了进一步深入研究数据统计随机

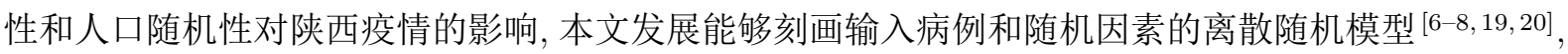
系统研究陕西省疫情的发展、控制策略的有效性, 分析由于逐步放宽出行限制、正常连续或间歇式人 口流动带来二次暴发的风险. 目前, COVID-19 在全球许多地区已经传播甚至暴发, 本文提出的模型思 想和研究方法为研究区域性疫情提供了方法和模型参考, 为公共卫生决策部门提供了有关旅行限制和 跟踪隔离等的决策信息.

\section{2 主要方法}

\section{1 数据}

我们从国家卫健委和陕西省卫健委官网获得了原始数据, 即陕西省 COVID-19 输入病例和确诊病 例数以及每一个确诊病例的详细文字记录 ${ }^{[5,16]}$. 陕西省实施的严格密切跟踪隔离措施, 提供了感染者 发病、首次就诊、入院和确诊的详细信息 (数据是匿名发布的). 数据不仅包括了新报告确诊病例数、 累计确诊病例数、累计治愈病例数、累计死亡病例数、累计疑似病例数, 也包括发病日期、首次就诊、 入院、实验室确认及出院或来陕日期 (输入病例) 等信息, 如图 1 所示. 根据详细记录得到了陕西省每 


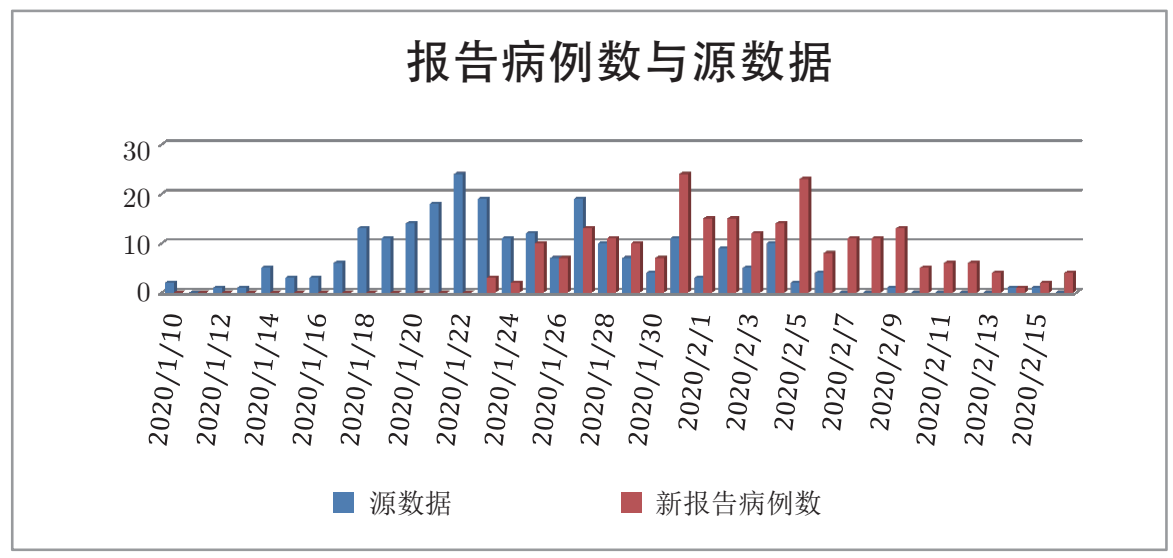

(a)

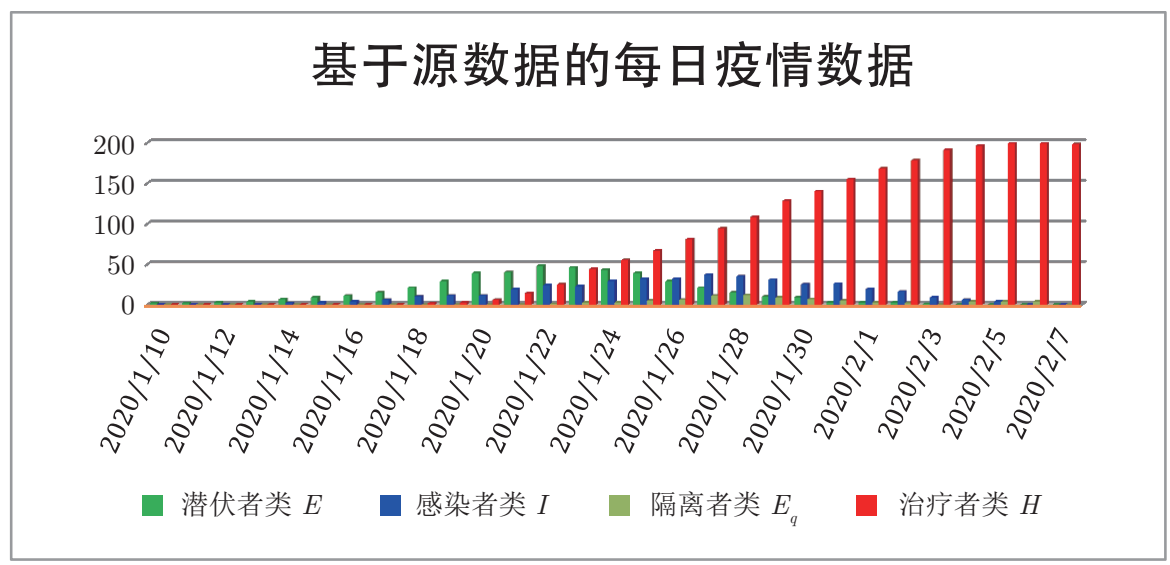

(b)

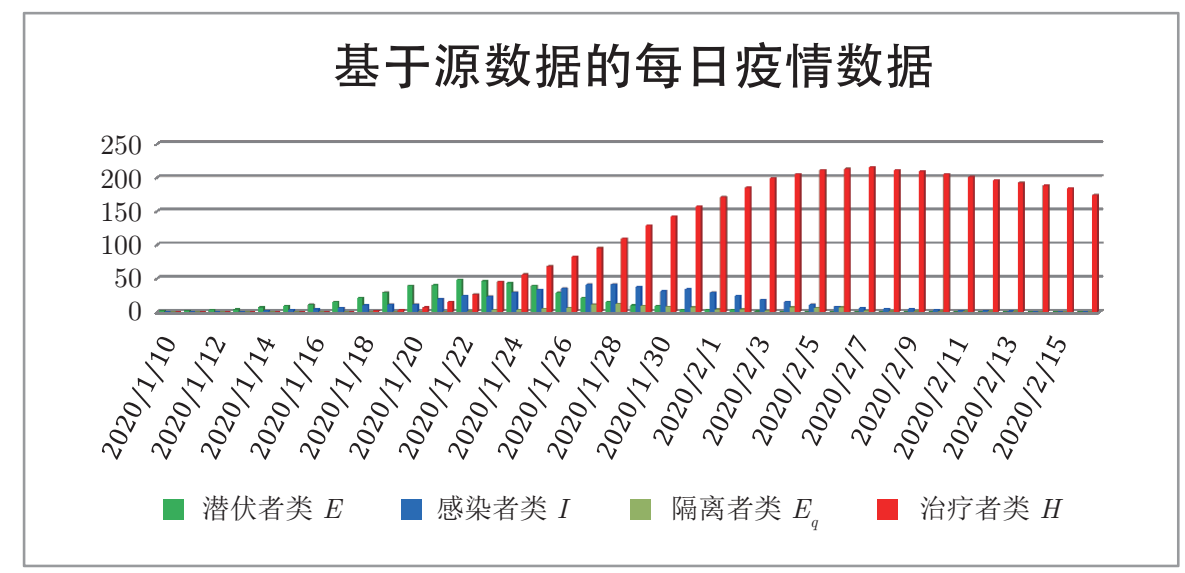

(c)

图 1 (网络版彩图) (a) 报告病例数与源数据; (b) 截至 2 月 9 日每日陕西省潜伏者类、感染者类和治疗者类的具 体数据; (c) 截至 2 月 16 日每日陕西省潜伏者类、感染者类和治疗者类的具体数据 
日潜伏者类、感染者类和治疗者类的具体数据, 称之为源数据, 如图 1(a)-1(c) 所示. 这里的每日治疗 者类病例数是指首次就诊然后被隔离, 而不是每日报道确诊的病例数. 此外, 我们还收集了百度迁徙 网站 (http://qianxi.baidu.com) 上的人口流动数据, 包含了 2020 年春节前和 2019 年春节后陕西省人 口流入的趋势, 如图 2 所示.

\section{2 有效再生数的估计}

用 $M_{t}$ 和 $C_{t}^{M}$ 分别表示陕西省第 $t$ 日新增输入病例数和新增确诊输入病例数, $T_{M}$ 表示输入病例 从输入到确诊的时长. 假设对 $k$ 个连续的日期 $j=t_{1}, \ldots, t_{k}, M_{j}$ 服从均值为 $\lambda_{j}$ 的 Poisson 分布, 其中

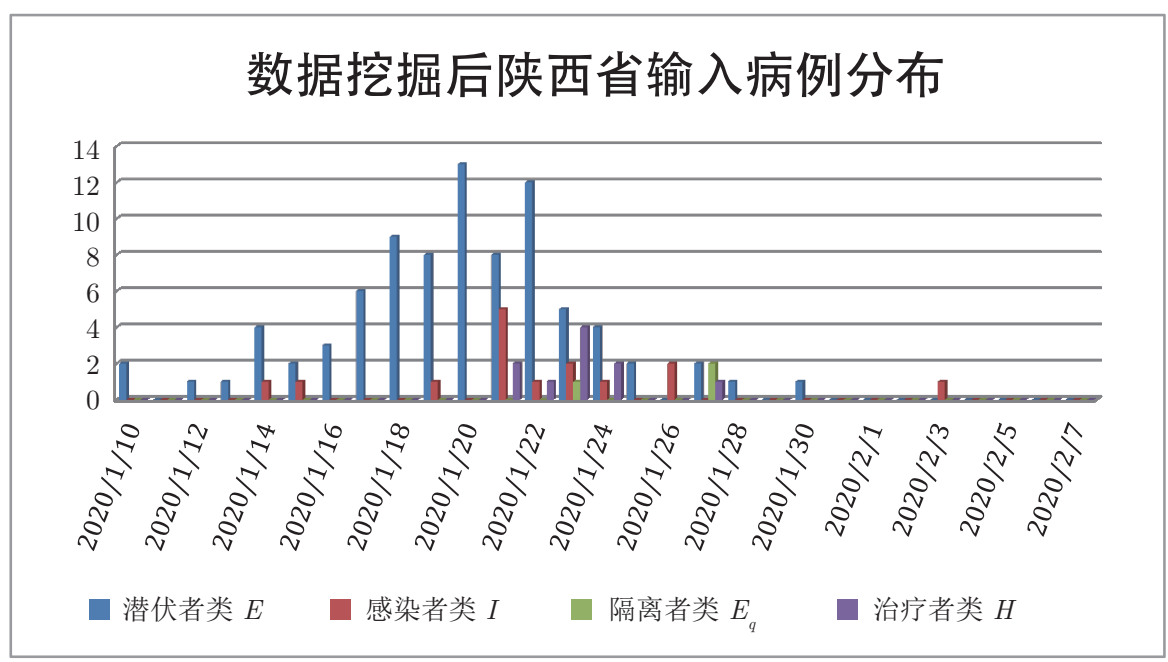

(a)

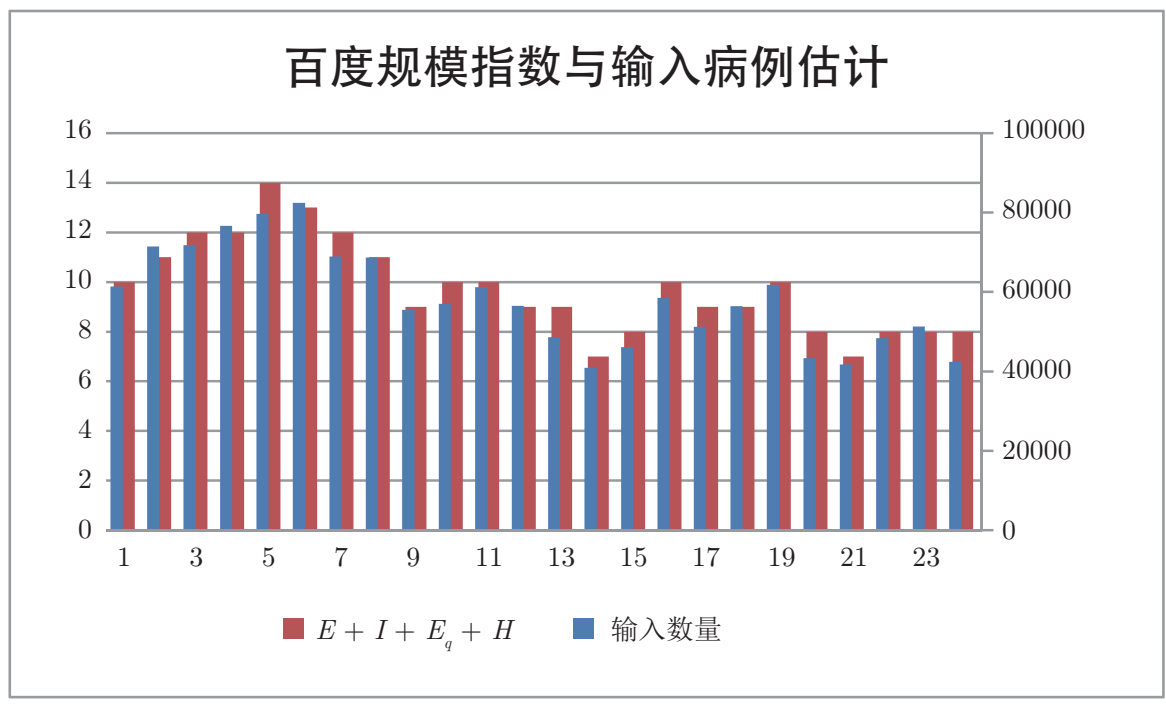

(b)

图 2 (网络版彩图) (a) 从 1 月 10 日到 2 月 7 日陕西省 4 类人群的输入病例数; (b) 陕西省每日输入病例数 $(2020$ 年 1 月 10 日到 2 月 2 日) 与百度规模指数 (2019 年 2 月 6 日至 3 月 1 日) 的关系 
$\lambda_{j}$ 是待估计的参数. 给定 $m$ 个连续的日期 $s_{1}, \ldots, s_{m}$ 内每日新增确诊输入病例数 $C_{s_{1}}^{M}, \ldots, C_{s_{m}}^{M}$ 和一 个输入病例在第 $j$ 日进入陕西省并在第 $i$ 日被确诊的概率 $p_{i j}=\mathrm{P}\left(i-j \leqslant T_{M}<i-j+1\right)$. 假设对任 意 $j, q_{j}=\sum_{i=\max \left\{j, s_{1}\right\}}^{s_{m}} p_{i j}>0$, 则可以使用逆卷积方法, 利用 $C_{s_{1}}^{M}, \ldots, C_{s_{m}}^{M}$ 和 $p_{i j}$ 对参数 $\lambda_{t_{1}}, \ldots, \lambda_{t_{k}}$ 加以估计. 我们使用 Richardson-Lucy 迭代算法 ${ }^{[21]}$ 求解这个问题, 即按照如下公式迭代更新未知参数 的估计值:

$$
C_{i}^{M(n)}=\sum_{j=t_{1}}^{i} p_{i j} \lambda_{j}^{(n)}, \quad \lambda_{j}^{(n+1)}=\frac{\lambda_{j}^{(n)}}{q_{j}} \sum_{i=\max \left\{j, s_{1}\right\}}^{s_{m}} \frac{p_{i j} C_{i}^{M}}{C_{i}^{M(n)}},
$$

其中 $C_{i}^{M(n)}$ 和 $\lambda_{j}^{(n)}$ 是第 $n$ 次迭代时 $C_{i}^{M}$ 和 $\lambda_{j}$ 的估计值. 迭代过程在拟合误差

$$
\chi^{2}=\frac{1}{m} \sum_{i=s_{1}}^{s_{m}} \frac{\left(C_{i}^{M(n)}-C_{i}^{M}\right)^{2}}{C_{i}^{M(n)}}
$$

尽可能小并且 $\lambda_{t_{1}}^{(n)}, \ldots, \lambda_{t_{k}}^{(n)}$ 取值合理的时候停止. 上述过程要求 $q_{j}>0$, 即在第 $j$ 日进入陕西省的输 入病例中至少有一部分会在 $s_{1}, \ldots, s_{m}$ 这段时期内被确诊. 因此在开始迭代前根据 $T_{M}$ 的分布来计算 $q_{j}$ 的值, 并选取适当的 $t_{1}$ 和 $t_{k}$ 使得 $q_{j}>0$ 对 $t_{1} \leqslant j \leqslant t_{k}$ 成立. 给定每日新增确诊本地病例数和本 地病例从被感染到确诊的时长的分布, 我们可以用相同的方法估计一段时期内每日新增本地感染者数 (将第 $t$ 日新增本地感染者数记为 $L_{t}$ ).

用 $T_{h}$ 表示输入病例从进入陕西省到感染本地易感者的时长, 将 $T_{h}$ 和续代时间 (serial interval) 的分布函数分别记为 $H(\tau)$ 和 $G(\tau)$. 为了在考虑输入病例的情况下估计有效再生数 $R(t)$, 我们推广文 献 $[17,18]$ 中的方法. 对 $R(t)$, 有 $\mathbb{E}\left[L_{t}\right]=R(t) \sum_{\tau=1}^{t-1}\left(g_{\tau} L_{t-\tau}+h_{\tau} M_{t-\tau}\right)$, 其中 $g_{\tau}=G(\tau)-G(\tau-1)$, $h_{\tau}=H(\tau)-H(\tau-1)$.

\section{3 离散随机模型}

基于疾病的传播机理、个体的流行病学状态及防控措施, 我们可将总人群分为易感者类 $(S)$ 、潜 伏者类 $(E)$ 、感染者类 $(I)$ 、住院者类 $(H)$ 、治愈者类 $(R)$ 、隔离的易感者类 $\left(S_{q}\right)$ 和隔离的疑似者类 (B) 7 个仓室, 流程如图 3 所示. 本文扩展了我们之前研究中的模型框架, 加入追踪隔离的疑似者类这 一仓室. 该仓室由追踪隔离的潜伏者和与该疾病所表现的临床症状相似的个体两部分组成. 考虑到早 期陕西省的输入病例数很小, 相比确定性模型, 带有随机输入的差分系统更适合刻画这一情况, 并能 很好地描述人口统计的随机性. 因此, 我们建立了如下的新模型 ${ }^{[19,20,22]}$ :

$$
\begin{aligned}
& S_{t+1}=S_{t}-D_{11}(t)-D_{12}(t)-D_{13}(t)+D_{51}(t)+(1-f) D_{41}(t), \\
& E_{t+1}=E_{t}+(1-q(t)) D_{11}(t)-D_{21}(t)+P_{E}\left(\lambda_{E}\right), \\
& I_{t+1}=I_{t}+D_{21}(t)-D_{31}(t)-D_{32}(t)-D_{33}(t)+P_{I}\left(\lambda_{I}\right), \\
& B_{t+1}=B_{t}+q(t) D_{11}(t)+D_{13}(t)-D_{41}(t)+P_{B}\left(\lambda_{B}\right), \\
& S_{q_{t+1}}=S_{q_{t}}+D_{12}(t)-D_{51}(t), \\
& H_{t+1}=H_{t}+D_{31}(t)+f D_{41}(t)-D_{61}(t)-D_{62}(t)+P_{H}\left(\lambda_{H}\right), \\
& R_{t+1}=R_{t}+D_{33}(t)+D_{61}(t),
\end{aligned}
$$




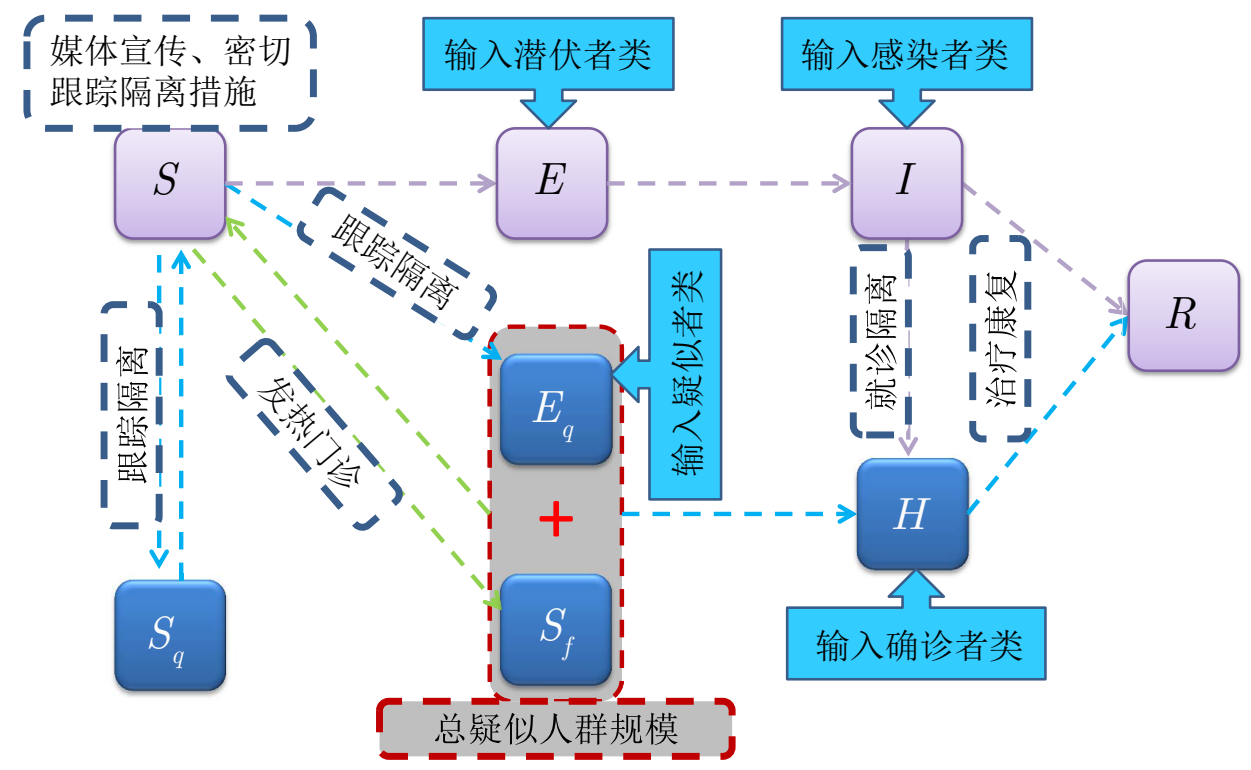

图 3 (网络版彩图) COVID-19 疫情与防控策略模型示意图. 防控策略包括密切跟踪隔离和医院隔离. 灰色区域表 示疑似病例和发热门诊患者

模型 (3.1) 中的随机变量服从参数为 $n$ 和 $p$ 的二项分布, 记为 $\operatorname{Bin}(n, p)$, 具体形式如下 (参见文 献 $[19,20])$ :

$$
\begin{aligned}
& D_{11}(t) \sim \operatorname{Bin}\left(S_{t}, P_{11}(t)\right), \quad D_{12}(t) \sim \operatorname{Bin}\left(S_{t}, P_{12}(t)\right), \\
& D_{13}(t) \sim \operatorname{Bin}\left(S_{t}, P_{13}\right), \quad D_{21}(t) \sim \operatorname{Bin}\left(E_{t}, P_{21}\right), \\
& D_{31}(t) \sim \operatorname{Bin}\left(I_{t}, P_{31}(t)\right), \quad D_{32}(t) \sim \operatorname{Bin}\left(I_{t}, P_{32}\right), \\
& D_{33}(t) \sim \operatorname{Bin}\left(I_{t}, P_{33}\right), \quad D_{41}(t) \sim \operatorname{Bin}\left(B_{t}, P_{41}\right), \\
& D_{51}(t) \sim \operatorname{Bin}\left(S_{q t}, P_{51}\right), \quad D_{61}(t) \sim \operatorname{Bin}\left(H_{t}, P_{61}\right), \\
& D_{62}(t) \sim \operatorname{Bin}\left(H_{t}, P_{32}\right),
\end{aligned}
$$

其中

$$
\begin{aligned}
& P_{11}(t)=1-\exp \left[-\beta c(t) \frac{I}{N} h\right], \\
& P_{12}(t)=1-\exp \left[-c(t) q(t)(1-\beta) \frac{I}{N} h\right], \\
& P_{13}=1-\exp (-m h), \quad P_{21}=1-\exp (-\sigma h), \\
& P_{31}(t)=1-\exp \left(-\delta_{I}(t) h\right), \quad P_{32}=1-\exp (-\alpha h), \\
& P_{33}=1-\exp \left(-\gamma_{I} h\right), \quad P_{41}=1-\exp (-b h), \\
& P_{51}=1-\exp (-\lambda h), \quad P_{61}=1-\exp \left(-\gamma_{H} h\right),
\end{aligned}
$$

这里 $c(t)$ 表示接触率函数, 函数形式见 (3.2); 常数 $m$ 表示通过普通临床诊断从易感者类到隔离的疑 
似者类的转化率. 若被确诊感染了 COVID-19, 则隔离的疑似者将有 $f$ 的比例以 $b$ 速率离开该仓室, 进 入住院者仓室, 而 $1-f$ 比例部分经隔离检验未被 COVID-19 感染, 则回到易感者仓室. 令疾病接触 时传播概率为 $\beta$, 接触数为 $c$. 通过密切追踪策略, 假设有 $q$ 比例的接触者被隔离, 并且依赖他们是否 感染分别以 $\beta c q$ 和 $(1-\beta) c q$ 的速率分别移动到仓室 $B$ 和 $S_{q}$, 而其他在追踪过程中被遗漏为 $1-q$ 比 例, 一旦被感染, 他们将以 $\beta c(1-q)$ 的速率移至潜伏者仓室 $E$, 否则仍留在仓室 $S$ 中. 表 1 给出所有 变量和参数的定义.

当每天输入潜伏者、感染者、疑似者和住院病例人数未知时, 可以通过 Poisson 分布来描述, 分别 记为 $P_{E}\left(\lambda_{E}\right) 、 P_{I}\left(\lambda_{I}\right) 、 P_{B}\left(\lambda_{B}\right)$ 和 $P_{H}\left(\lambda_{H}\right)$, 其中参数为 $\lambda_{E} 、 \lambda_{I} 、 \lambda_{B}$ 和 $\lambda_{H}$. 具体形式如下:

$$
\begin{array}{ll}
P_{E}\left(E_{t}=m\right)=\frac{\lambda_{E}^{m} \mathrm{e}^{-\lambda_{E}}}{m !}, & P_{I}\left(I_{t}=m\right)=\frac{\lambda_{I}^{m} \mathrm{e}^{-\lambda_{I}}}{m !}, \\
P_{B}\left(B_{t}=m\right)=\frac{\lambda_{B}^{m} \mathrm{e}^{-\lambda_{B}}}{m !}, & P_{H}\left(H_{t}=m\right)=\frac{\lambda_{H}^{m} \mathrm{e}^{-\lambda_{H}}}{m !} .
\end{array}
$$

\begin{tabular}{|c|c|c|c|}
\hline 参数 & 定义 & 陕西省 & 来源 \\
\hline$c_{0}$ & 初始接触率 & 15 & 文献 [8] \\
\hline$c_{b}$ & 当前控制策略下的最小接触率 & 6.0371 & 参数估计 \\
\hline$\gamma_{1}$ & 接触率的指数递减速率 & 0.051 & 参数估计 \\
\hline$\beta$ & 每次接触传播的概率 & 0.21 & 参数估计 \\
\hline$q_{0}$ & 最初潜伏者的隔离率 & 0 & 数据 \\
\hline$q_{m}$ & 在当前控制策略下潜伏者的最大隔离率 & 0.5228 & 参数估计 \\
\hline$\gamma_{2}$ & 潜伏者隔离率的指数增长速率 & 0.0799 & 参数估计 \\
\hline$m$ & 易感者向疑似者的转移率 & 0.007 & 参数估计 \\
\hline$b$ & 疑似者的检出率 & 0.0397 & 参数估计 \\
\hline$f$ & 确诊比例: 疑似的潜伏者向隔离的感染者的转移率 & 0.01 & 参数估计 \\
\hline$\sigma$ & 潜伏者到感染者的转移率 & $1 / 2.38$ & 数据 \\
\hline$\lambda$ & 隔离的未受感染接触者释放回社区的速率 & $1 / 12$ & 文献 [8] \\
\hline$\delta_{I 0}$ & 有症状的感染者向隔离的感染者的初始转化率 & $1 / 4.01$ & 数据 \\
\hline$\delta_{I f}$ & 最快诊断速度 & 0.4076 & 参数估计 \\
\hline$\gamma_{3}$ & 诊断率的指数递减速率 & 0.0832 & 参数估计 \\
\hline$\gamma_{I}$ & 感染者的恢复率 & 0.0497 & 文献 [8] \\
\hline$\gamma_{H}$ & 隔离感染者的恢复率 & 0.0199 & 参数估计 \\
\hline$\alpha$ & 因病死亡率 & 0 & 数据 \\
\hline 初值 & 定义 & 陕西省 & 来源 \\
\hline$S(0)$ & 易感者的初值 & $9.9895 \times 10^{4}$ & 文献 $[16]$ \\
\hline$E(0)$ & 潜伏者的初值 & 0 & 数据 \\
\hline$I(0)$ & 感染者的初值 & 0 & 数据 \\
\hline$B(0)$ & 疑似者的初值 & 0 & 数据 \\
\hline$S_{q}(0)$ & 隔离的易感者的初值 & 0 & 数据 \\
\hline$H(0)$ & 隔离的感染者的初值 & 0 & 数据 \\
\hline$R(0)$ & 恢复者的初值 & 0 & 数据 \\
\hline
\end{tabular}

表 1 陕西省 COVID-19 疫情的参数估计 
自 2020 年 1 月 23 日在武汉实施封城策略以来, 其他省份的预防策略也不断得到加强. 为了真实 地描述持续加强的控制策略和不断提高的诊断率, 假设接触率、隔离率、诊断率和治愈率为时间 $t$ 的 函数. 假设接触率 $c(t)$ 是关于时间 $t$ 的递减函数, 具有形式如下:

$$
c(t)= \begin{cases}c_{0}, & 2020 \text { 年 } 1 \text { 月 } 23 \text { 日之前, } \\ \left(c_{0}-c_{b}\right) \mathrm{e}^{-r_{1} t}+c_{b}, & \text { 其他, }\end{cases}
$$

其中 $c_{0}$ 表示初始时间段 (即 2020 年 1 月 23 日之前) 的接触率, $c(0)=c_{0} ; c_{b}$ 表示当前控制策略下接 触率的最小值, 即 $\lim _{t \rightarrow \infty} c(t)=c_{b}$ 且 $c_{b}<c_{0} ; r_{1}$ 表示接触数指数下降的速率.

同样, 定义隔离率 $q(t)$ 为关于时间 $t$ 的递增函数, 记为

$$
q(t)= \begin{cases}q_{0}, & 2020 \text { 年 } 1 \text { 月 } 23 \text { 日之前, } \\ \left(q_{0}-q_{m}\right) \mathrm{e}^{-r_{2} t}+q_{m}, & \text { 其他, }\end{cases}
$$

其中 $q_{0}$ 表示潜伏个体的初始隔离率; $q_{m}$ 表示当前控制策略下的最大隔离率, 即 $\lim _{t \rightarrow \infty} q(t)=q_{m}$ 且 满足 $q_{m}>q_{0} ; r_{2}$ 表示隔离率的指数增长率. 此外, 转移率 $\delta_{I}(t)$ 也定义为时间 $t$ 的递增函数, 因此, 检 测周期 $1 / \delta_{I}(t)$ 是时间 $t$ 的递减函数, 其形式如下:

$$
\frac{1}{\delta_{I}(t)}= \begin{cases}\frac{1}{\delta_{I 0}}, & 2020 \text { 年 } 1 \text { 月 } 23 \text { 日之前, } \\ \left(\frac{1}{\delta_{I 0}}-\frac{1}{\delta_{I f}}\right) \mathrm{e}^{-r_{3} t}+\frac{1}{\delta_{I f}}, & \text { 其他, }\end{cases}
$$

其中 $\delta_{I 0}$ 表示初始确诊率, $\delta_{I}(0)=\delta_{I 0} ; \delta_{I f}$ 表示最快的确诊率, 即 $\lim _{t \rightarrow \infty} \delta_{I}(t)=\delta_{I f}$ 且满足 $\delta_{I 0}<\delta_{I f}$; $r_{3}$ 表示诊断时间的指数减少率. 设 $T_{c}$ 为 2020 年 1 月 23 日开始实施严格控制措施的时间点. 直到 2020 年 2 月 4 日, 陕西省尚无治愈病例, 因此, 2020 年 2 月 4 日之前治愈率 $\gamma_{H}$ 为 0 . 自 2020 年 2 月 7 日以来, 患者治愈的数量迅速增加. 因此, 基于实际情况, 治愈率被定义为分段常数函数.

\section{4 主要结论}

\section{1 数据挖掘}

基于数据信息可知每个感染者的状态演化和空间分布, 得到潜伏者类、感染者类和治疗者类的人 数, 图 4 显示了陕西省疫情的演变过程. 图 1(a) 给出了每日新增报告病例数和源数据中的每日新增 病例数, 两个时间序列的比较表明源数据中的新病例比新报告病例数据提前约 7 天. 特别是, 图 1(b) 和 1 (c) 分别给出了 2 月 7 日和 2 月 15 日前确诊的病例在各个仓室的分布情况. 陕西省于 2020 年 1 月 23 日开始公布病例数, 但第一例输入潜伏病例早在 2020 年 1 月 10 日就来到陕西省, 而且大部 分输入的潜伏期病例在 2020 年 1 月底前进入了陕西省 (如图 2(a) 所示). 图 2(b) 显示了输入病例总 数与流入陕西省的人口数量之间的相关关系, 据此我们可以计算流入人口中的感染病例比例. 进一步, 根据源数据可以得到输入病例 (圆形) 和本地病例 (方形) 的空间分布和疾病的演变. 从图 4 可以看出 大多数病例在西安市, 少数病例在陕北地区, 而相对较多的病例在与湖北省相邻的陕南地区.

根据陕西省报告的所有病例的源数据，我们使用 Kaplan-Meier 方法估计从发病到首次就诊 (用 $T_{2}^{W}$ 表示) 的中位持续时间约为 0.60 天, 从首次就诊 (隔离) 到确诊 (用 $T_{3}^{W}$ 表示) 的中位持续时间约 
2020 年 1 月 10 日

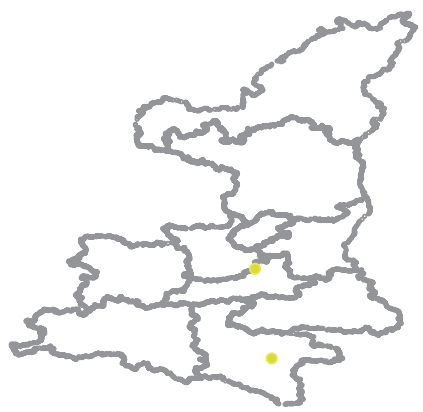

2020 年 1 月 21 日

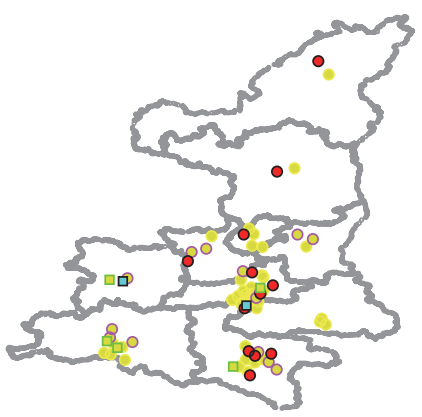

2020 年 2 月 1 日

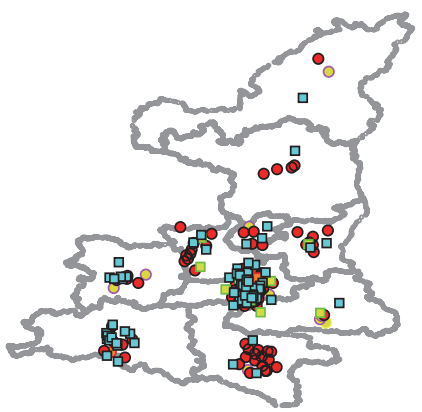

2020 年 1 月 14 日

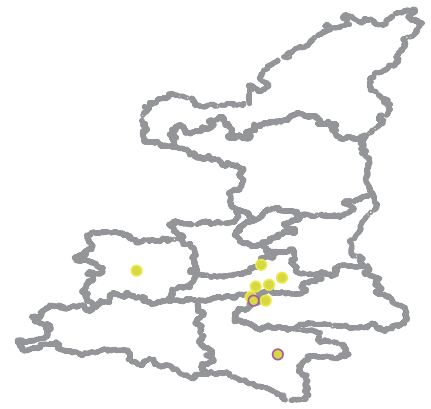

2020 年 1 月 24 日

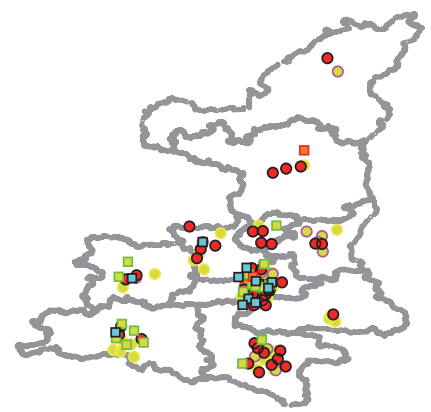

2020 年 2 月 4 日

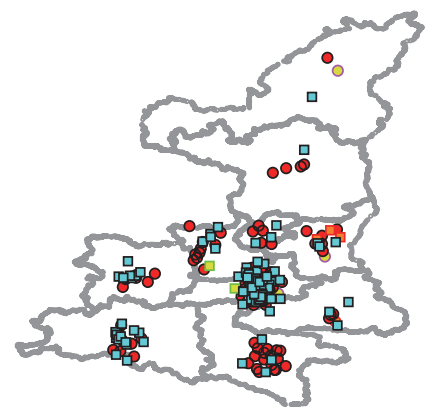

2020 年 1 月 18 日

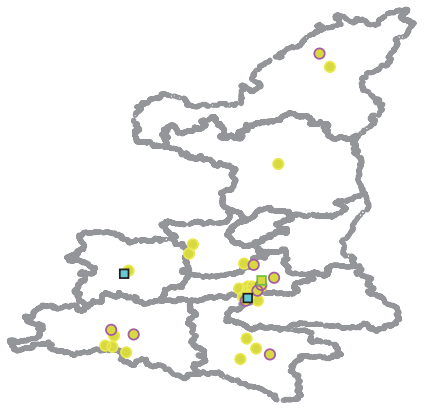

2020 年 1 月 28 日

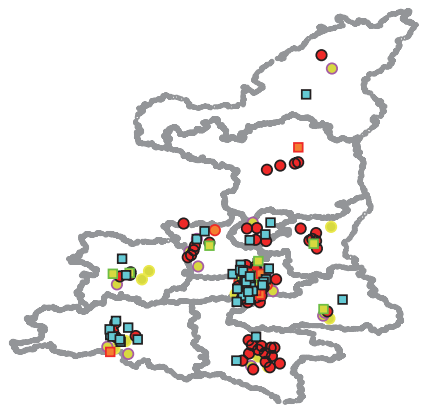

2020 年 2 月 7 日

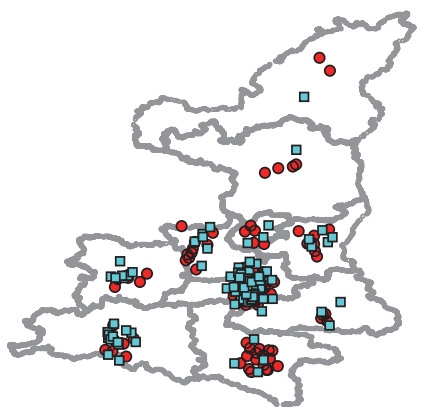

图 4 (网络版彩图) 基于源数据得到的输入病例 (圆圈) 和本地病例 (方块) 的空间分布和时间演化. 输入病例中各 颜色分别表示: 潜伏者类 (黄色)、感染者类 (洋红色)、隔离者类 (红色) 和治疗者类 (黑色); 本地病例: 感染者类 (绿色)、隔离者类 (红色) 和治疗者类 (黑色)

为 3.43 天 (如图 5 所示). 特别地, 对于输入病例, 从输入到发病 (用 $T_{1}^{I}$ 表示) 的中位持续时间约为 2.38 天, 最长潜伏期为 19 天; 从发病到首次就诊 (用 $T_{2}^{I}$ 表示) 的中位持续时间约为 1 天, 最长 (短) 持续时间为 15 (0) 天; 从首次就诊到确诊 (用 $T_{3}^{I}$ 表示) 的中位持续时间约为 3.05 天, 最长 (短) 持续 时间为 13 (1) 天. 对于本地病例, 从发病到首次就诊 (用 $T_{2}^{L}$ 表示) 的中位时间约为 0.29 天, 最长 (短) 感染时间为 $10(0)$ 天; 从首次就诊到确诊 (用 $T_{3}^{L}$ 表示) 的中位持续时间约为 3.9 天, 最长 (短) 持续时 间为 16 (1) 天. 上述分析表明这些持续时间比湖北省数据显示的持续时间要短 (参见文献 $[1,11-13]$ ), 这表明陕西省实施的控制措施较为及时. 


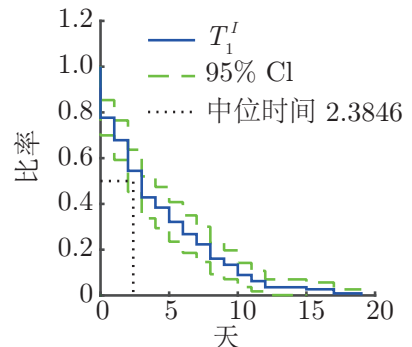

(a)

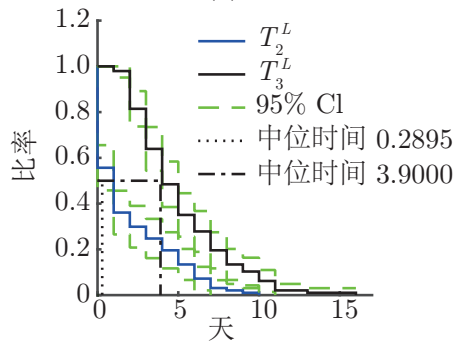

(d)

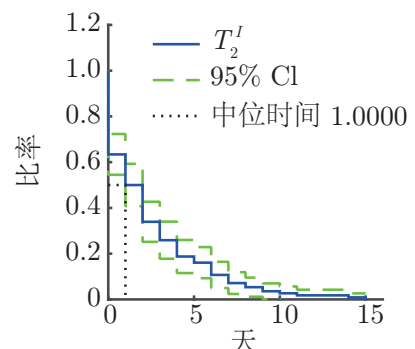

(b)

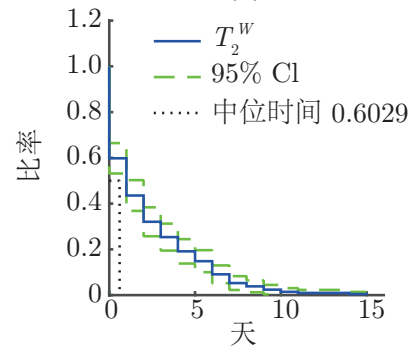

(e)

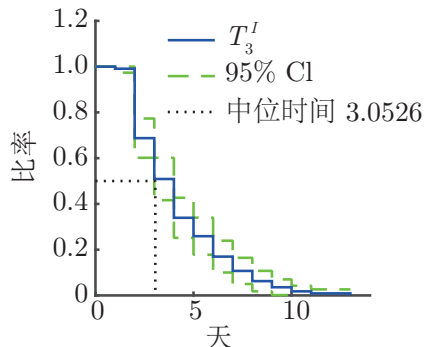

(c)

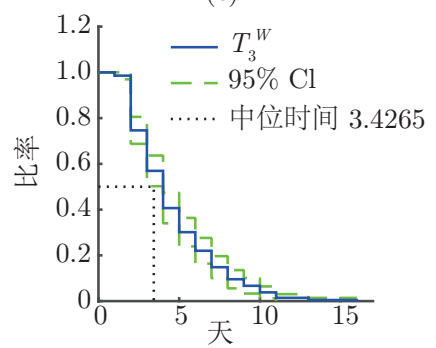

(f)

图 5 (网络版彩图) 使用 Kaplan-Meier 方法估计陕西省输入病例中从输入到发病的时长 (a), 记为 $T_{1}^{I}$; 从发病 到首次就诊的时长 (b), 记为 $T_{2}^{I}$; 从首次就诊到确诊的时长 (c), 记为 $T_{3}^{I}$. 陕西省本地病例中从发病到首次就诊、从 首次就诊到确诊的时长 $(\mathrm{d})$, 分别记为 $T_{2}^{L}$ 和 $T_{3}^{L}$. 陕西省所有病例中从发病到首次就诊的时长 $(\mathrm{e})$, 记为 $T_{2}^{W} ;$ 从 首次就诊到确诊的时长 (f), 记为 $T_{3}^{W}$

\section{2 控制再生数和有效再生数}

从源数据中可以找到 21 条传播链 (感染树), 共涉及 60 个病例 (其中包括 13 个输入病例), 每条 传播链中患者的感染时间和二代感染者都是已知的. 这 21 个传播链中的首个病例所感染的二代病例 数分别为 $2 、 2 、 1 、 5 、 1 、 0 、 2 、 2 、 2 、 1 、 1 、 1 、 2 、 1 、 2 、 1 、 1 、 1 、 1 、 1 、 1$. 由此计算出控制再生数的均值 和标准差分别为 1.48 和 0.98 . 陕西省早期报告的病例以输入病例为主, 这些病例大多在 2020 年 2 月 3 日之前被确诊. 考虑在 2020 年 1 月 23 日至 2 月 3 日的受感染情况, 我们得到了 13 条传播链 (即 图 6 中的第 1-13 号感染链), 其控制再生数的均值和标准差分别为 1.69 和 1.18 .

在估计新增感染时, 我们需要用到输入病例从输入到确诊的时长 (记为 $T_{M}$ ) 和本地病例从感染到 确诊的时长 (记为 $T_{L}$ ) 的概率分布, 假设 $T_{M}$ 和 $T_{L}$ 服从 Weibull 分布. 我们利用 2020 年 2 月 16 日 前确诊的输入病例估计出 $T_{M}$ 的均值和标准差分别为 10.68 和 4.95 天. $T_{L}$ 等于潜伏期 $T_{L 1}$ 与本地病 例从发病到确诊的时长 $T_{L 2}$ 之和, 由文献 [2-4,12-14,23], 取 $T_{L 1}$ 的均值和标准差分别为 5.2 和 3.91 天, 利用 2020 年 2 月 16 日前确诊的本地病例的详细信息, 估计出 $T_{L 2}$ 的均值和标准差分别为 7.28 和 3.89 天, 进而可以计算得到 $T_{L}$ 的均值和标准差分别为 12.48 和 5.52 天. 然后, 我们使用逆卷积方法, 基于 2020 年 2 月 16 日前每日报告确诊病例数及 $T_{M}$ 和 $T_{L}$ 的概率分布, 估计出了陕西省 2020 年 2 月 12 日前每日新增输入病例数和每日新增本地感染者数 (统一称之为每日新增感染者数), 如图 7(a) 所示. 从陕西省源数据中, 我们获得了部分病例的感染时间, 由此可以得到 2020 年 2 月 16 日前的每 日新增感染者数, 如图 7(b) 所示, 从图中可以看出实际源数据汇总的新增感染人数和估计的每日新增 感染人数的趋势是吻合的, 但是数量少. 这是因为有些确诊病例的感染时间未知, 故并未纳入新增感 染人数中.

为了估计有效再生数, 取续代时间的概率分布 $g_{\tau}$ 是均值为 7.5 天、标准差为 3.4 天的 Gamma 分 布 ${ }^{[4,13]}$. 因为输入病例从进入陕西省到感染本地病例的时长的分布 $h_{\tau}$ 未知, 首先取 $h_{\tau}=g_{\tau}$ 来估计 

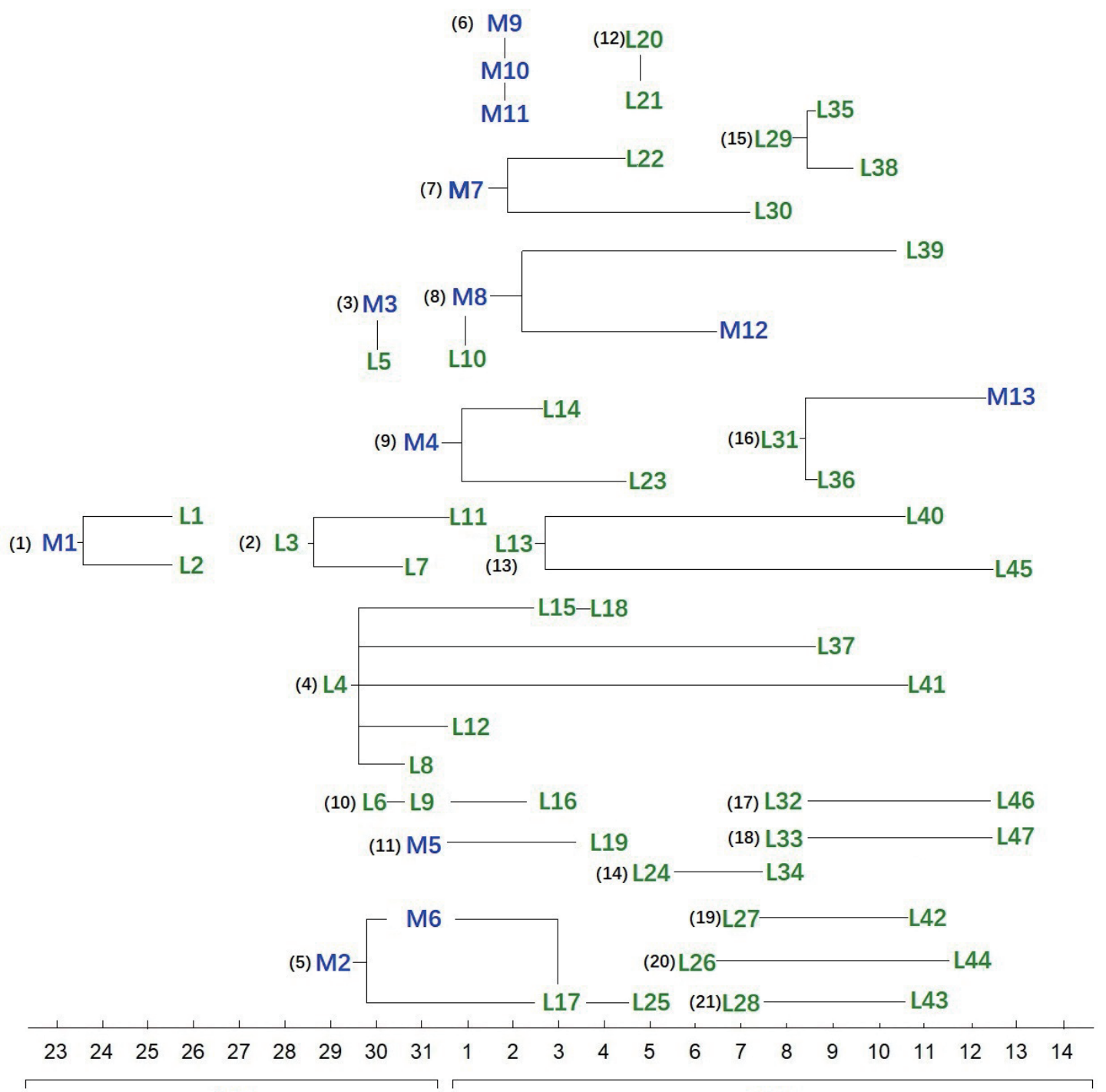

1月

2月

图 6 (网络版彩图) 传播链. 蓝色的 $\mathrm{M} i$ 代表输入病例, 绿色的 $\mathrm{L} j$ 代表本地病例, $i$ 和 $j$ 代表患者编号

有效再生数 $R(t)$, 结果如图 7(c) 所示. 从图中可以看出有效再生数在 2020 年 1 月 23 日开始下降, 在 1 月 27 日下降到 1 以下, 并稳定在 0 附近. 为了研究 $T_{h}$ 变化对有效再生数的影响, 我们通过改变 $T_{h}$ 的均值来实施敏感性分析, 如图 7(d) 所示. 从图中可以看出 $T_{h}$ 的均值越大, 有效再生数 $R(t)$ 取值大 于 1 的时间就越长, 且上升并超过 1 的时间也越早.

\section{3 人口流动对疫情的影响}

首先基于最小二乘法, 通过 1,000 次离散随机模型拟合 2020 年 1 月 10 日至 2 月 7 日的源数据 (图 8 中带点的紫色曲线) 并估计参数, 求均值得到未知参数 (表 1 中列出), 随后利用表 1 中的参数得 到模型 (3.1) 的 500 条随机解, 如图 8(a)-8(c) 所示. 图 8(d) 显示估计的易感人群变化较为显著. 另外, 


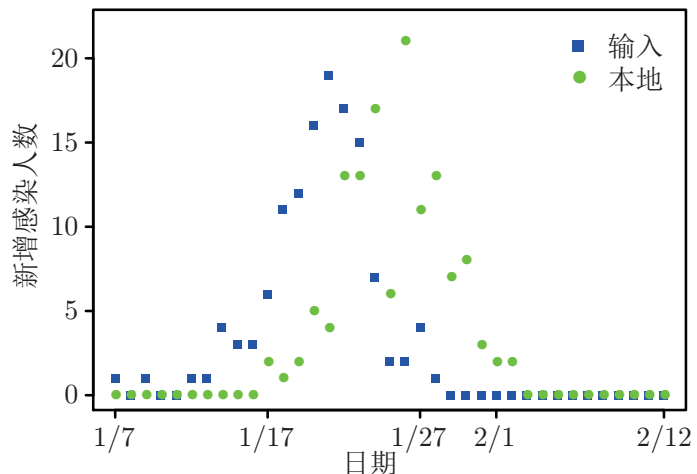

(a)

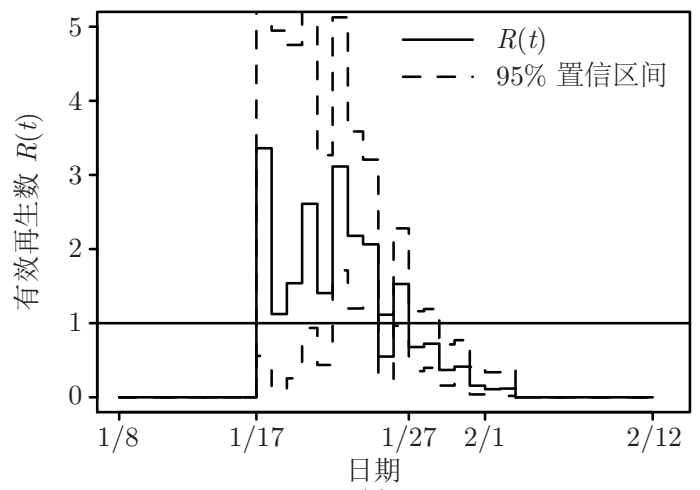

(c)

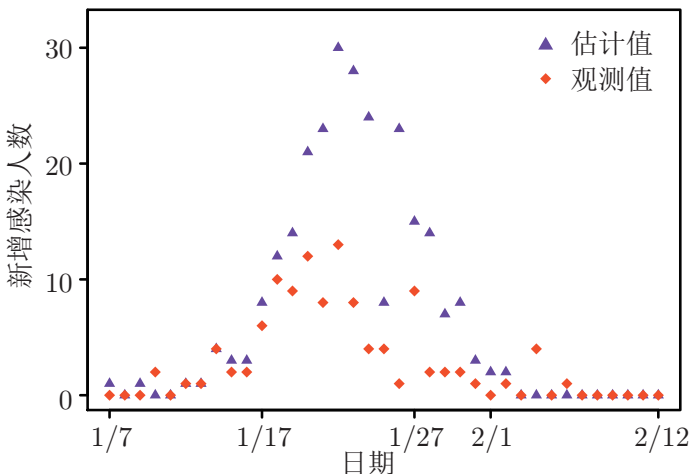

(b)

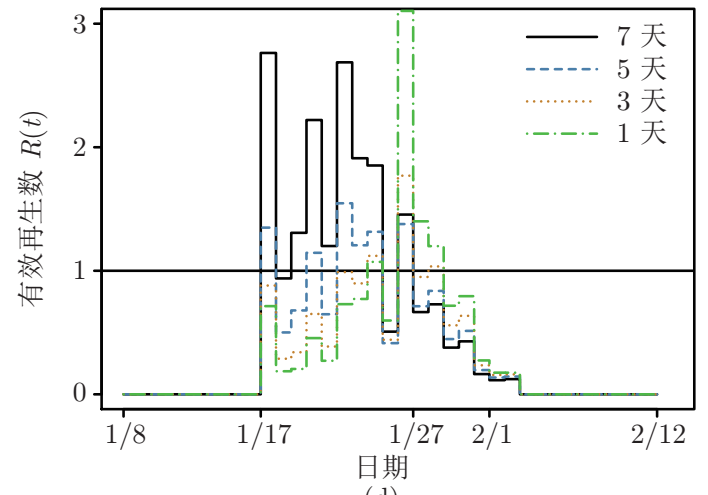

(d)

图 7 (网络版彩图) (a) 基于报告确诊数估计的新增输入和本地感染者数; (b) 新增感染者数的估计值与真实值的比 较; (c) 和 (d) 有效再生数 $R(t)$

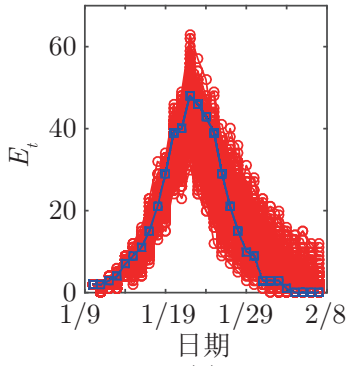

(a)

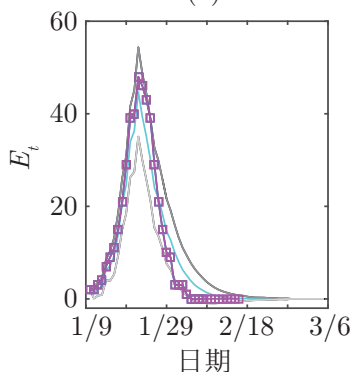

(e)

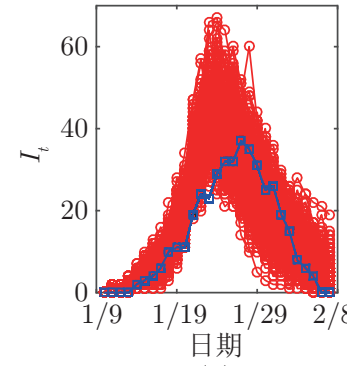

(b)

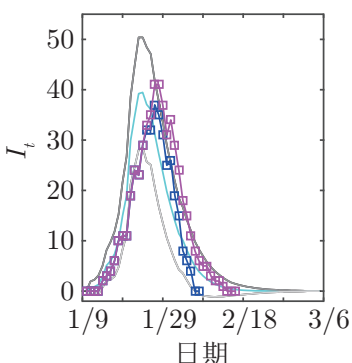

(f)

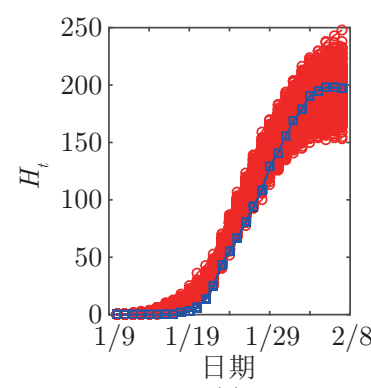

(c)

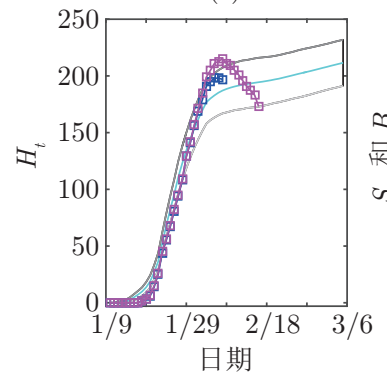

(g)

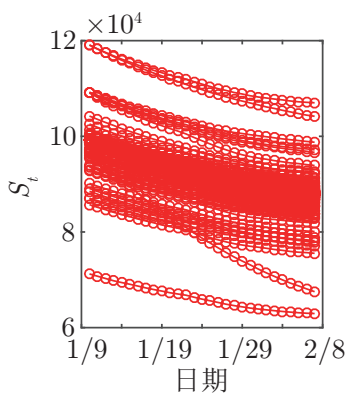

(d)

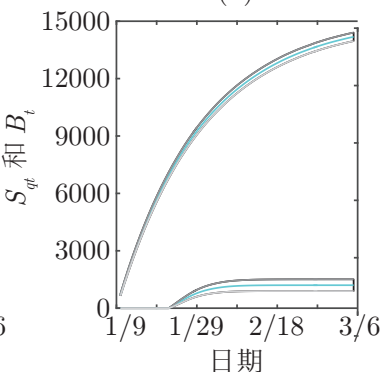

(h)

图 8 (网络版彩图) (a)-(c) 500 次模拟的数据拟合 $(E(t) 、 I(t)$ 和 $H(t)) ;(\mathrm{d})$ 易感人群数量的变化; $(\mathrm{e})-(\mathrm{g})$ 平 均潜伏者类 $E(t)$ 、感染者类 $I(t)$ 和治疗者类 $H(t)$ 随时间的变化; (h) 隔离的易感者类 $S_{q}(t)$ 和总疑似者类 $B(t)$ 随时间的变化 (绿色曲线表示平均, 灰色曲线表示 $95 \%$ 置信区间) 
估计的潜伏者类 $E_{t}$ 、感染者类 $I_{t}$ 和治疗者类 $H_{t}$ 的均值 (绿色曲线) 及其 $95 \%$ 的置信区间 (CI) 如 图 8(e)-8(g) 所示. 由于在 2020 年 2 月 4 日才有首例患者治愈出院, 估计的恢复率 $\gamma_{H}$ 非常小以致 于治疗者类 $H_{t}$ 的平均值持续缓慢增加 (如图 8(g) 所示). 基于 2020 年 1 月 10 日至 2 月 16 日的数 据 (带有圆点的紫色曲线) 对模型进行验证, 发现源数据落在了 $E_{t} 、 I_{t}$ 和 $H_{t}$ 等估计值的 $95 \%$ 置信区 间内.

分析复工、复学引起人口流动对疫情的影响, 特别是诱发二次暴发的可能性, 将为决策部门提供 定量的决策依据. 在模拟人口流动时, 假设陕西省恢复正常秩序后的人口流动趋势与 2019 年同期相 同. 令 2020 年 1 月 17 日至 1 月 22 日期间陕西省 $E 、 I 、 E_{q}$ 和 $H 4$ 类人口流入率的平均比例分别为 $p_{E} 、 p_{I} 、 p_{B}$ 和 $p_{H}$, 它们可以通过输入病例数和人口流动性来计算得到. 假设恢复正常工作后陕西省 $E 、 I 、 E_{q}$ 和 $H 4$ 类人口流入率的平均比例分别为 $\hat{p}_{E} 、 \hat{p}_{I} 、 \hat{p}_{B}$ 和 $\hat{p}_{H}$. 假设 $\hat{p}_{E} 、 \hat{p}_{I} 、 \hat{p}_{B}$ 和 $\hat{p}_{H}$ 的初始 值与 $p_{E} 、 p_{I} 、 p_{B}$ 和 $p_{H}$ 相同, 由此得到同时段的输入病例数. 当 2 月 24 日开始恢复正常工作后, 考 虑疾病发展越来越弱的情况, 假设比例 $\hat{p}_{E} 、 \hat{p}_{I} 、 \hat{p}_{B}$ 和 $\hat{p}_{H}$ 减少 $30 \% 、 60 \%$ 甚至 $90 \%$.

我们通过输入病例数据和从其他城市迁入陕西省的人口数据来预测疾病感染人数. 假设从 2020 年 2 月 24 日到 3 月 9 日, 人群开始持续地返回陕西省, 从图 9(a) 和 9 (b) 可以看出, 会出现一次较 大规模的二次暴发. 如果输入病例比例下降 30\%、60\% 和 $90 \%$, 则二次暴发的规模将越来越小, 如 图 9(c)-9(d)、9(e)-9(f) 和 9(g)-9(h) 所示. 进一步, 如果将流入模式改为间歇性流入, 即允许人群每隔 一周间歇性地进入陕西省, 从图 10 可以看出将有两个较小的二次暴发. 减少输入病例的比例会大幅 度降低疫情再次暴发的峰值. 对比图 9 和 10 可知, 间歇式、错峰人口迁入模式同时加强密切跟踪隔离 措施 (降低输入病例的比例), 不可能诱发较大的二次暴发, 是复工、复学的较好选择.

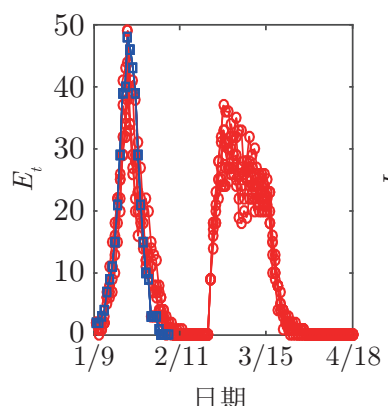

(a)

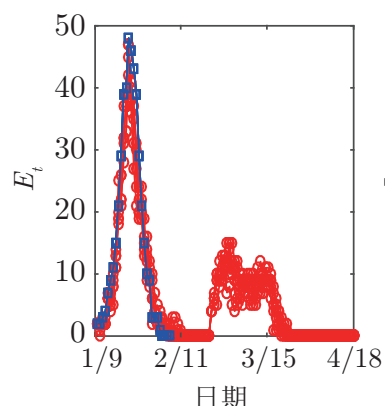

(e)

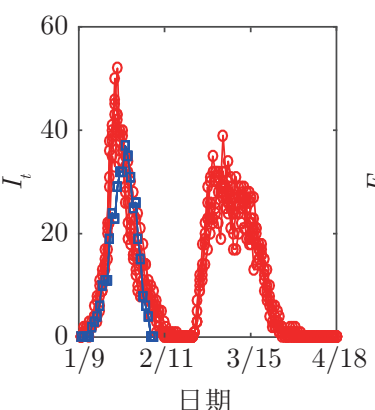

(b)

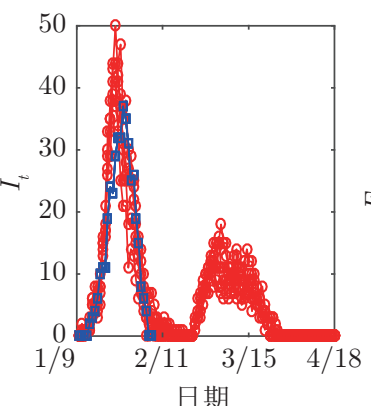

(f)

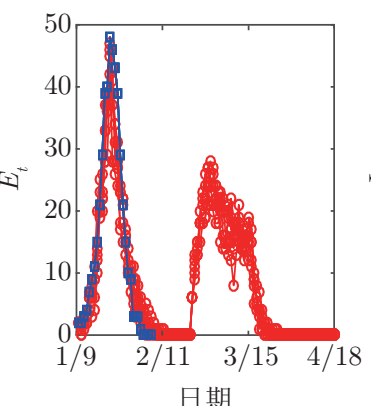

(c)

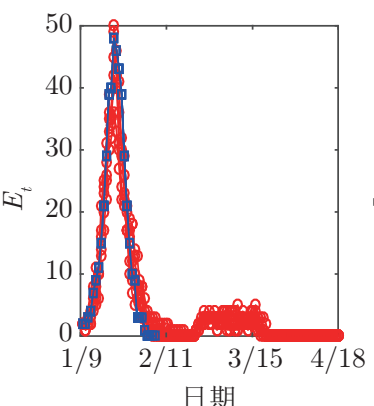

(g)

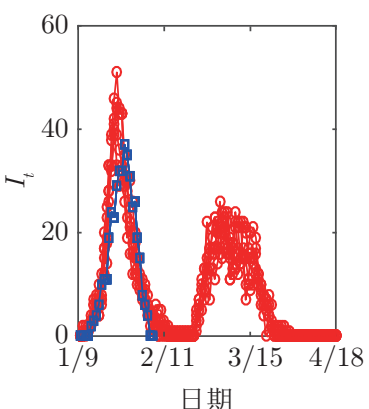

(d)

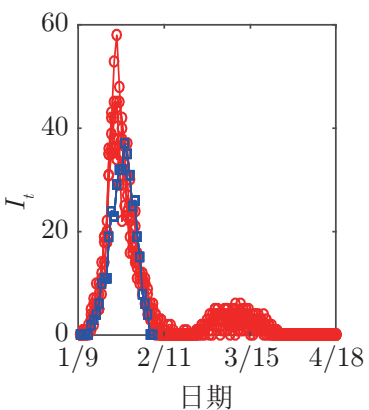

(h)

图 9 (网络版彩图) 连续的人口流入对潜伏者类和感染者数量的影响. (a) 和 (b) 为基线参数值; (c) 和 (d) 流入 减少基线值的 $30 \%$; (e) 和 (f) 流入减少基线值的 $60 \%$; (g) 和 (h) 流入减少基线值的 $90 \%$. 这里的人口流入持 续了 24 天 (从 2020 年 2 月 24 日到 3 月 18 日) 


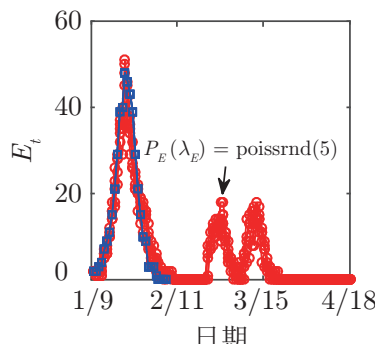

(a)

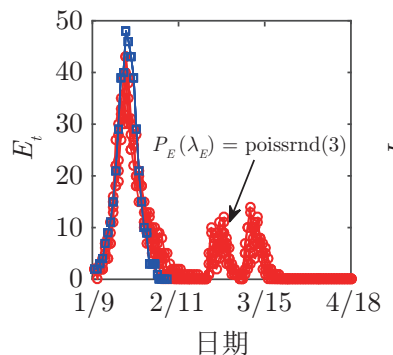

(e)

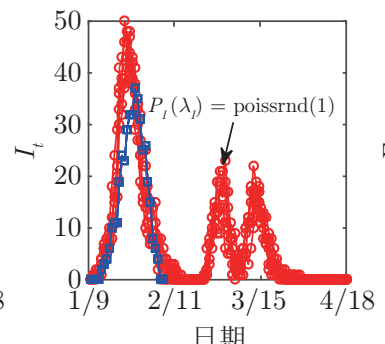

(b)

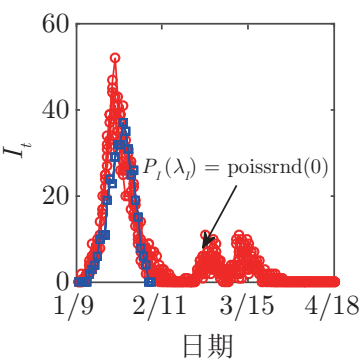

(f)

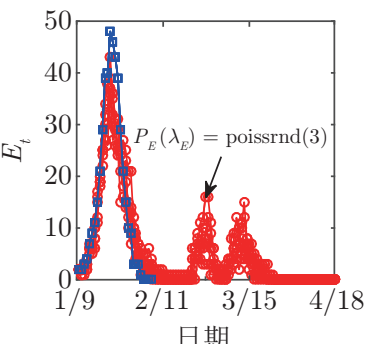

(c)

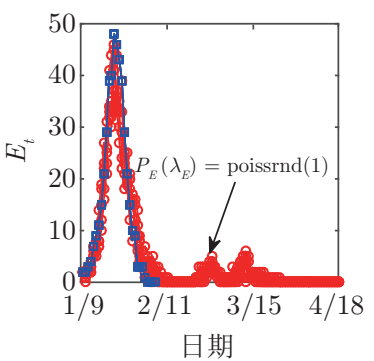

(g)

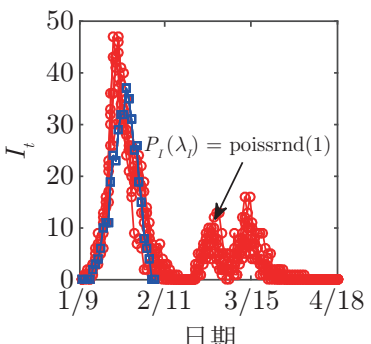

(d)

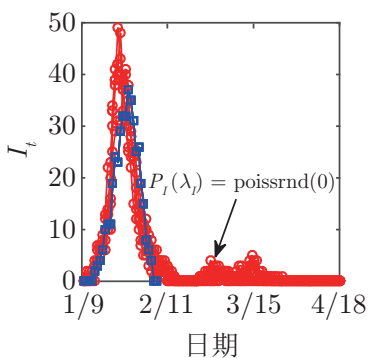

(h)

图 10 (网络版彩图) 间歇性的人口流入对潜伏者类和感染者数量的影响. (a) 和 (b) 为基线参数值; (c) 和 (d) 流 入减少基线值的 $30 \%$; (e)-(f) 流入减少基线值的 $60 \%$; (g)-(h) 流入减少基线值的 $90 \%$. 这里在 2020 年 2 月 24 日后的第一周 (2 月 24 日至 3 月 1 日) 和第三周 (3 月 9 日至 15 日) 有人口流入, 第二周和第四周以及随后 的几周停止

\section{5 讨论与结论}

陕西省卫健委每天都会公布详细的疫情情况, 输入病例报告了具体的来陕时间, 除每天新报告 (或 累计) 确诊病例数外, 记录不仅包括患者发病、首次就诊、入院和确诊的具体时间, 而且还有详细的感 染过程, 即什么时候谁感染了谁等. 因而可以得到潜伏期、感染期和住院期的分布, 从而可以给出陕西 省疫情的演变过程. 数据分析揭示了陕西省病例从发病到首次就诊, 到住院治疗, 再到确诊的时间都 比我国整体或湖北省相应病例的时间间隔要短 (参见文献 $[1,11-13]$ ), 这表明陕西省实施的控制措施较 为及时, 控制力度大. 从源数据中可以得到新冠肺炎在陕西省传播的感染链, 从而能够计算出在实施 严格控制策略下的平均控制再生数. 利用逆卷积方法和续代时间的分布可以估计有效再生数, 结论显 示有效再生数自 2020 年 1 月 23 日以来一直在下降, 目前稳定且几乎为 0 , 这与最近一段时间没有新 确诊病例数或确诊病例数非常少吻合.

为了刻画相对较少的病例数, 而且病例中大约一半是输入病例以及由此带来的随机性, 我们建立 了具有随机输入的离散系统, 该模型框架克服了确定性模型不能刻画输入病例及随机性 ${ }^{[6-8,24]}$ 的严重 不足. 基于源数据得到了潜伏者类 $(E)$ 、感染者类 $(I)$ 和治疗者类 $(H)$ 的队列数据以及感染者状态转 移的空间分布, 据此能较为准确地估计模型参数. 我们利用陕西省与其他城市的人口流动数据, 研究 了人口流动 (复工) 对疫情的二次暴发的影响. 主要结果表明: 在输入病例比例较少的情况下, 保持间 歇性的人口流入, 尽管会导致小规模的二次暴发, 但传播风险低.

本文中提出的随机离散模型及其分析方法, 可作为研究陕西省或其他类似省份 (病例数不多, 输 入病例占较大比例) 疫情发展趋势的重要模型框架. 但这类模型框架的一个缺点是, 很难从理论方面 系统研究其阈值理论, 而在不考虑随机输入时, 针对这类随机差分系统的理论分析是有可能的, 这为 
我们后期研究提供了思路. 最后强调: 使用源数据而不是简单地用每日报告确诊病例数或累计数据来 研究 COVID-19 的流行情况是非常重要的, 这些源数据不仅可以为准确评价湖北省外其他地区的疫情 提供重要的数据信息, 而且克服了报告的延迟, 并为评价不同地区公共卫生干预策略的有效性和时效 性提供重要而准确的数据支持.

\section{参考文献}

1 Cohen J, Normile D. New SARS-like virus in China triggers alarm. Science, 2020, 367: 234-235

2 Guan W, Ni Z, Hu Y, et al. Clinical characteristics of 2019 novel coronavirus infection in China. MedRxiv, 2020, doi: 10.1101/2020.02.06.20020974

3 Heymann D L, Shindo N. COVID-19: What is next for public health? Lancet, 2020, 395: 542-545

4 Li Q, Guan X, Wu P, et al. Early transmission dynamics in Wuhan, China, of novel coronavirus-infected pneumonia. N Engl J Med, 2020, 382: 1109-1207

5 中华人民共和国国家卫生健康委员会. 疫情通报. Http://www.nhc.gov.cn, 2020

6 Tang B, Bragazzi N L, Li Q, et al. An updated estimation of the risk of transmission of the novel coronavirus (2019-nCov). Infect Dis Model, 2020, 5: 248-255

7 Tang B, Xia F, Tang S, et al. The evolution of quarantined and suspected cases determines the final trend of the 2019-nCoV epidemics based on multi-source data analyses. Available at SSRN: Https://ssrn.com/abstract=3537099, 2020

8 Tang B, Wang X, Li Q, et al. Estimation of the transmission risk of the 2019-nCoV and its implication for public health interventions. J Clin Med, 2020, 9: 462

9 Zhao S, Lin Q, Ran J, et al. Preliminary estimation of the basic reproduction number of novel coronavirus (2019-nCoV) in China, from 2019 to 2020: A data-driven analysis in the early phase of the outbreak. Int J Infect Dis, 2020, 92: $214-217$

10 Zhao S, Zhuang Z, Cao P, et al. Quantifying the association between domestic travel and the exportation of novel coronavirus (2019-nCoV) cases from Wuhan, China in 2020: A correlational analysis. J Travel Med, 2020, 27: taaa022

11 Boldog P, Tekeli T, Vizi Z, et al. Risk assessment of novel coronavirus COVID-19 outbreaks outside China. J Clin Med, 2020, 9: 571

12 Du Z, Wang L, Cauchemez S, et al. Risk for transportation of coronavirus disease from Wuhan to other cities in China. Emerg Infect Dis, 2020, 26: 1049-1052

13 Gilbert M, Pullano G, Pinotti F, et al. Preparedness and vulnerability of African countries against importations of COVID-19: A modelling study. Lancet, 2020, 395: 871-877

14 Sun K, Chen J, Viboud C. Early epidemiological analysis of the coronavirus disease 2019 outbreak based on crowdsourced data: A population-level observational study. Lancet Digital Health, 2020, doi: 10.1016/S2589-7500(20)30026-1

15 Xiao Y, Tang S, Wu J. Media impact switching surface during an infectious disease outbreak. Sci Rep, 2015, 5: 7838

16 中华人民共和国陕西省卫生健康委员会. 疫情通报. Http://sxwjw.shaanxi.gov.cn/col/col863/index.html, 2020

17 Nishiura H. Correcting the actual reproduction number: A simple method to estimate $\mathrm{R}_{0}$ from early epidemic growth data. Int J Environ Res Public Health, 2010, 7: 291-302

18 Nishiura H, Chowell G. The effective reproduction number as a prelude to statistical estimation of time-dependent epidemic trends. In: Mathematical and Statistical Estimation Approaches in Epidemiology. Dordrecht: Springer, 2009, 103-121

19 Lekone P E, Finkenstädt B F. Statistical inference in a stochastic epidemic SEIR model with control intervention: Ebola as a case study. Biometrics, 2006, 62: 1170-1177

20 Mode C J, Sleeman C K. Stochastic Processes in Epidemiology. Singapore: World Scientific, 2000

21 Goldstein E, Dushoff J, Ma J, et al. Reconstructing influenza incidence by deconvolution of daily mortality time series. Proc Natl Acad Sci USA, 2009, 106: 21825-21829

22 王霞, 唐三一, 陈勇, 等. 新型冠状病毒肺炎疫情下武汉及周边地区何时复工? 数据驱动的网络模型分析. 中国科 学: 数学, 2020, 50: 969-978

23 Parry J. China coronavirus: Cases surge as official admits human to human transmission. BMJ, 2020, 368: m236

24 Keeling M J, Rohnai P. Modeling Infectious Diseases in Humans and Animals. Princeton: Princeton University Press, 2008 


\title{
Analysis of COVID-19 epidemic traced data and stochastic discrete transmission dynamic model
}

\author{
Sanyi Tang, Biao Tang, Nicola Luigi Bragazzi, Fan Xia, Tangjuan Li, Sha He, Pengyu Ren, Xia Wang, \\ Changcheng Xiang, Zhihang Peng, Jianhong Wu \& Yanni Xiao
}

\begin{abstract}
The epidemic of novel coronavirus pneumonia has spread throughout the country. The early epidemic cases in many provinces, including Shaanxi, are mainly imported cases. The latest epidemic situation has been decreasing under restrict prevention and control strategies. Accessing the efficacy of control measures, analyzing the impact of population flow on the epidemic situation are of great significance for the study of the epidemic situation in Shaanxi (or other areas with imported cases as the main cases) and the future response to emergent infectious diseases. According to the detailed data published by Shaanxi, we can obtain the transmission chains (infection tree), and the median durations from the illness onset to the first medical visit, to the admission, and then to the final confirmation. We can obtain the daily number of latent, infectious and hospitalized individuals and the spatial distribution of their state evolution. The control reproduction number of COVID-19 epidemic was determined (1.48-1.69). We develop the statistical inference method to calculate the effective regeneration number under the strict control measures in Shaanxi province. Furthermore, a novel stochastic discrete transmission model for COVID-19 was proposed, which integrates possible interventions and import cases. The parameterization of the formulated model was realized through multiple source data. Our main conclusion shows that intermittent population flow, close attention and effective isolation of the floating population can effectively reduce the risk of secondary outbreak, which consequently provides decision support for the orderly organization of returning to work/school.
\end{abstract}

Keywords COVID-19, case importation, stochastic discrete model, effective reproduction number, population flow

MSC(2010) 34F05, 65N21

doi: $10.1360 /$ SSM-2020-0053 